A N N A L E S Annales de Bretagne et des Pays de l'Ouest

\title{
Michel PERTUE (dir.), Suffrage, citoyenneté et révolutions (1789-1848),
}

\section{Serge Bianchi}

\section{(2) OpenEdition}

1 Journals

Édition électronique

URL : http://journals.openedition.org/abpo/1316

DOI : $10.4000 /$ abpo.1316

ISBN : 978-2-7535-1493-5

ISSN : 2108-6443

Éditeur

Presses universitaires de Rennes

Édition imprimée

Date de publication : 20 mars 2004

Pagination : 159-161

ISBN : 978-2-86847-976-1

ISSN : 0399-0826

\section{Référence électronique}

Serge Bianchi, « Michel PERTUE (dir.), Suffrage, citoyenneté et révolutions (1789-1848), », Annales de Bretagne et des Pays de l'Ouest [En ligne], 111-1 | 2004, mis en ligne le 20 mars 2006, consulté le 23 septembre 2020. URL : http://journals.openedition.org/abpo/1316 ; DOI : https://doi.org/10.4000/ abpo. 1316 
La Révolution hideuse de l'an II ne trouve grâce que dans l'héroïsme des soldats et des marins, et indirectement dans les atrocités que l'auteur attribue aux Vendéens et aux futurs "terroristes blancs " de l'an III, ce qui autorise une conclusion mettant en parallèle les principes de 1789 et l'hécatombe qui aurait suivi la chute de la monarchie.

Certes, le livre est daté (les années 1830) d'une époque qui faisait le procès de la Terreur et, en partie, l'apologie des Girondins. Il ne saurait évidemment reprendre les acquis récents de l'historiographie qui disqualifieraient tous les derniers chapitres. Mais c'est par le talent et certaines intuitions de l'auteur qu'il devient dangereux pour un lecteur non informé. Ce dernier aura tendance à accepter en bloc un récit qui véhicule les pires préjugés et parti pris sur la période montagnarde et sans-culotte de la Convention. Qui rappellera que les représentants ne sont pas des proconsuls sanglants (Michel Biard), que l'égalitarisme sans-culotte se construit sur un idéal des droits naturels et sociaux (Albert Soboul), que la justice de l'an II n'est pas le vertige d'arbitraire décrit dans ces pages, que le "vandalisme " n'a pas abattu les châteaux et les églises, que les spéculateurs n'ont pas été décimés pendant la Terreur, que les mouvements paysans ont longtemps été " révolutionnaires " (Anatoli Ado), et qu'une partie des villes et campagnes bretonnes ont été " bleues " pendant toute la décennie (acquis du bicentenaire)? Faute de cette clairvoyance élémentaire, et au delà même de ce souffle si contagieux qui anime le livre, ces Mémoires risquent de contribuer à figer les mythes et légendes qui façonnent aujourd'hui l'opinion publique sur la nature de la Révolution. Débutée à Rennes dans l'agitation des jeunes gens (Moreau en vedette) et l'invention de la presse politique, la Révolution, devenue bourgeoise, serait devenue de plus en plus antipaysanne et centralisatrice, pour culminer dans la chasse aux suspects, la fureur sanguinaire des représentants en mission et des "tyrans en sabots ", le délire antireligieux et le fanatisme des " tricoteuses". Ainsi s'ancrerait une lecture bas-bretonne spécifique de la marche de la Révolution, complaisante à la pire des légendes noires du processus républicain. Reste alors une dernière question : doit-on conseiller la lecture des Mémoires, pour leurs qualités littéraires et psychologiques? Ou mettre en garde contre les déformations systématiques d'une partie de ce réquisitoire? L'aperçu proposé ici ne vise qu'à donner aux éventuels lecteurs quelques clés qui nous ont paru salutaires, pour juger ensuite sur pièce, en leur âme et conscience...

Serge BIANCHI

PERTUE, Michel (dir.), Suffrage, citoyenneté et révolutions (1789-1848), Société des études robespierristes, 2002, 181 p., $25 €$.

Dans la collection Études révolutionnaires, paraît le numéro 3, actes d'une journée d'études de mars 2001 où s'est noué un " dialogue d'historiens, politologues et juristes ", autour des questions du suffrage et de la "mise en place d'une démocratie représentative " (Michel Pertué) entre la Révolution française et la Seconde République. C'est dire l'originalité d'une démarche réunissant des spécialistes des questions électorales, autour des expériences successives d'approfondissement du droit de vote, comparant des régimes aussi différents - et jusque-là traités de façon autonome - que sont la Révolution montagnarde, thermidorienne, le Consulat et l'Empire, la restauration et la monarchie censitaire. 
Il manquerait l'esprit électoral de 1848, présent toutefois dans une synthèse iconographique suggestive de Michel Offerlé. L'apport d'une telle manifestation peut être dégagé de deux manières complémentaires. D'une part, résumer brièvement les acquis des contributions successives et la fécondité de leurs approches. De l'autre, peser les continuités et ruptures, les espaces de comparaison, les nouveautés décelables dans la synthèse délicate que tente Raymond Huard, rapporteur tout désigné pour sa familiarité avec les questions du suffrage universel.

Les problématiques choisies par les six orateurs et oratrice paraissent a priori peu conciliables. Serge Aberdam traite de la capacité délibérative des assemblées primaires, au moment des ratifications référendaires des constitutions de 1793 et de l'an III. Cette pratique n'est ni isolée, ni réductible à la " démocratie pure " (le terme " démocratie directe " serait anachronique), mais renvoie à des dispositions de la constitution de l'an I et débouche sur un ensemble de vœux et de délibérations, portées à Paris par des "envoyés du souverain "à l'été 1793. L'an VIII met fin à cette permanence des capacités délibératives d'assemblées, qui seraient théoriquement vouées à la seule élection dans le cadre d'un système rigoureusement représentatif. Bernard Gainot étudie les débats autour de l'organisation du calendrier et du rituel électoral sous le Directoire. Démocrates et " censitaires " s'affrontent autour des conditions du vote, de l'utilité des listes de candidats, des cérémonies susceptibles de rendre plus efficace le mode de consultation et plus satisfaisants les résultats pour les tenants du pouvoir central. Philippe Tanchoux tente d'évaluer, dans un exposé très structuré, la part de continuité (des principes) et d'innovation (des procédures) introduites par le Consulat et l'Empire par rapport à la " révolution électorale " de 1789-1790. Si les assemblées primaires sont théoriquement maintenues, le processus délibératif est disloqué au bénéfice des comportements individuels, des candidatures officielles et des campagnes d'opinion réalisées sous la tutelle administrative de pouvoirs centraux, voire du détournement arithmétique des résultats. Malcolm Crook prouve le caractère décisif, délibératif et organisé des assemblées électorales sous la monarchie censitaire. D'une part, les électeurs censitaires seraient plus nombreux que les électeurs de la Révolution française. De l'autre, ce suffrage (en assemblée d'arrondissement ou de département) fait l'objet d'une participation remarquable, à la mesure de campagnes d'opinion menées par la hiérarchie administrative et par une opposition plus structurée, voire plus populaire qu'on ne l'a affirmé jusqu'à ce jour. Anne Verjus montre, de façon subtile, que la Restauration a élargi un espace censitaire réputé étroit, par une législation " familialiste " permettant au citoyen d'adjoindre dans la déclaration des contributions les revenus de son épouse et de ses ascendants. Cette particularité permet une extension du droit de vote à des petits propriétaires, à des gendres, dans une logique libérale qui disparaitra par la suite. Michel Offerlé permet, en 55 illustrations, de cerner les représentations et les enjeux autour de l'acte d'élire et de délibérer, entre la Seconde République et la France de Vichy. Chacune des communications, tirée d'une thèse ou d'un ouvrage récent, contribue à une tenue d'ensemble particulièrement riche et ...foisonnante. Il reste à marquer les connections et les avancées d'un chantier collectif en pleine mutation.

Certains domaines ont été négligés, soit sciemment, car traités dans d'autres ouvrages ou journées d'étude - le Guide pour la recherche ou les actes de la journée de Dijon, Voter et élire à l'époque contemporaine parus en 1999 -; soit par défaut, comme la participation électorale, la réception publique de l'élargissement ou du rétrcissement par les législateurs du doit de vote. D'autres ne per- 
mettent guère de recoupements directs, comme le dossier iconographique ou l'analyse " familialiste " du droit de vote. Les aspects techniques et parlementaires l'emportent dans plusieurs interventions, permettant de poser la question de l'encadrement du vote en assemblée au gré des régimes et des logiques qui se sont succédés, en particulier pour le rôle fondamental du bureau, élu ou nommé. La mise en perspective des capacités de délibération en assemblée est un des aspects les plus riches de la journée, sans que toutes les voies aient pu en être explorées. Les calendriers électoraux, l'individualisation progressive des procédures dans le maintien d'un cadre collectif, la place des rituels et des solennités dans les moments électoraux ont nourri d'autres réflexions transversales. Raymond Huard montre avec érudition comment de telles problématiques s'insèrent dans un approfondissement historiographique quantitatif, qualitatif et géographique des études électorales depuis une vingtaine d'années dont il pointe les avancées et les zones d'ombre. Il tente d'inscrire les contenus de la journée dans cette perspective ample. Il reste que de telles rencontres peuvent souffrir de la relative spécialisation des contributions et que les raccordements entre les régimes et les pratiques relèvent parfois de la virtuosité intellectuelle. Les espaces (canton, arrondissement, département), les enjeux, les publics, les législations défient souvent l'analyse comparative. Une autre démarche serait de réunir des spécialistes autour d'une thématique commune, diachronique, pour cerner avec plus d'efficacité les évolutions, les correspondances et les ruptures entre ces régimes qui ont expérimenté tant de modalités et de procédures électorales, du suffrage " large » de 1789 au suffrage universel masculin du printemps 1848.

Serge BIANCHI

Sonnet, Martine, Keriven, Brigitte, GHiati, Claude et HAVElAnge, Isabelle, Bibliographie annuelle de l'Histoire de France, t. 48, Année 2002, Paris, CNRS éditions, 2003.

La quarante-huitième livraison de la Bibliographie annuelle de l'Histoire de France est parue en novembre 2003. Les ingénieurs du CNRS chargés de son élaboration ont recensé dans ce volume 13465 références provenant principalement du dépouillement de 1382 revues françaises et 620 revues étrangères ainsi que de 314 ouvrages collectifs (Mélanges, Actes de colloques etc.) concernant l'Histoire de France, de la fin du v ${ }^{\mathrm{e}}$ siècle à 1958. Les chercheurs concernés sont toujours attentifs à la parution de cet indispensable instrument de travail. Le triple index, chronologique, par matières (incluant tous les noms de personnes et de lieux cités) et par noms d'auteurs facilite l'accès aux références. Celles-ci sont réparties selon un plan de classement thématique familier aux utilisateurs de la $B A H F$ (" manuels généraux et sciences auxiliaires de l'histoire ", " histoire politique ", " histoire des institutions ", " histoire économique ", " histoire sociale ", " histoire religieuse ", " histoire de la France outre-mer ", " histoire de la civilisation ", " histoire locale ").

Dans un bref mais très dense "Avertissement " liminaire, les auteurs présentent les statistiques qui ressortent du croisement des grandes périodes historiques et des principaux objets d'études. Évidemment, il ne s'agit que d'un aperçu qui doit être relativisé, mais les tableaux présentés ici ("périodes étudiées ", " histoire politique ", " champs historiographiques ") reflètent néanmoins l'évolution de la recherche au cours de ces dernières années (1998-2002) ou 